Meta

Journal des traducteurs

Translators' Journal

\title{
Rime et allitération dans les langues française et anglaise
}

\section{Henri Van Hoof}

Volume 53, numéro 4, décembre 2008

URI : https://id.erudit.org/iderudit/019654ar

DOI : https://doi.org/10.7202/019654ar

Aller au sommaire du numéro

Éditeur(s)

Les Presses de l'Université de Montréal

ISSN

0026-0452 (imprimé)

1492-1421 (numérique)

Découvrir la revue

Citer cet article

Hoof, H. V. (2008). Rime et allitération dans les langues française et anglaise. Meta, 53(4), 899-906. https://doi.org/10.7202/019654ar

\section{Résumé de l'article}

Étude de la rime et de l'allitération en français et en anglais. Petit retour historique sur les origines latines possibles et aux ressources mnémotechniques dans les formes rimées des proverbes et dictions ainsi que des allitérations. Analyse de quelques dictons météorologiques dans les deux langues et de locutions avec formes rimées et allitérées, ainsi que d'exclamations, apostrophes et jurons. 


\title{
ÉTUDES TERMINOLOGIQUES ET LINGUISTIQUES
}

\section{Rime et allitération dans les langues française et anglaise}

\author{
HENRI VAN HOOF \\ Centre de Terminologie de Bruxelles, Bruxelles, Belgique
}

\begin{abstract}
RÉSUMÉ
Étude de la rime et de l'allitération en français et en anglais. Petit retour historique sur les origines latines possibles et aux ressources mnémotechniques dans les formes rimées des proverbes et dictions ainsi que des allitérations. Analyse de quelques dictons météorologiques dans les deux langues et de locutions avec formes rimées et allitérées, ainsi que d'exclamations, apostrophes et jurons.
\end{abstract}

\begin{abstract}
This is a study of rhyme and alliteration in French and English. We review their possible historical origins in Latin, and consider the mnemonic resources contained in rhymed forms of proverbs, other sayings and alliterations. We then analyze a few meteorological sayings in both languages, idioms with rhymed and alliterative forms, and finally exclamations, apostrophes, and swearwords.
\end{abstract}

\section{MOTS-CLÉS/KEYWORDS}

rime, proverbes, dictons, assonance, allitération

Si l'on en croit les diverses hypothèses émises, la rime trouverait son origine dans les vers latins, qui faisaient rimer ensemble les deux hémistiches. Déplacée à la fin des vers, elle apparaît dans la poésie classique chrétienne, notamment dans les Instructiones adversus gentium deos, ouvrage en hexamètres du plus ancien poète chrétien, Commodien de Gaza (III ${ }^{\mathrm{e}}$ siècle), dans saint Augustin (IV ${ }^{\mathrm{e}}$ siècle), et persiste ensuite dans les hymnes rythmiques de l'Église catholique. À partir du Ix ${ }^{e}$ siècle, elle devient un élément fondamental de la poésie française, mais sous forme d'assonance, rime imparfaite reposant seulement sur l'identité de la dernière voyelle tonique. Et ce n'est qu'à partir du XII-XIII ${ }^{\mathrm{e}}$ siècle que la rime véritable triomphe. Chez les anciens peuples du Nord, c'est aussi l'assonance qui, avec l'allitération, a formé la base de la versification dans les poésies scandinaves et germaniques, et l'une comme l'autre sont toujours bien présentes dans la poésie anglaise des temps modernes.

Rime, assonance et allitération remplissent plusieurs fonctions: elles concourent à marquer un rythme, contribuent à l'harmonie, aident à mieux fixer les idées et à retenir les connaissances renfermées dans les vers. Si cela est vrai pour la poésie, ce l'est tout autant pour d'autres domaines. La langue juridique, par exemple, a toujours été riche en formules rimées ou allitératives (en foi de quoi/in witness whereof, etc.) 
et les trois procédés se rencontrent abondamment dans les proverbes et dictons, les comptines, les jurons, etc. Aussi nous proposons-nous d'en examiner les traces dans les créations de l'imaginaire collectif français et anglais, en nous limitant toutefois essentiellement à la rime et à l'allitération.

\section{PROVERBES ET DICTONS}

\section{Formes rimées}

Dans les créations de la sagesse populaire, la rime sert principalement de moyen mnémotechnique. En français, cette rime est parfois riche: comparaison n'est pas $\underline{\text { raison, }}$ patience passe science, l'intention vaut l'action, qui n'a point d'ami ne vit qu'à demi (avec allitération et assonance en plus), coucher de poule et lever de corbeau écartent l'homme du tombeau, etc., mais le plus souvent ne l'est pas: bien faire et laisser braire, bouche de miel cœur de fiel, l'occasion fait le larron, qui vole un œuf vole un bœuf, qui va à la chasse perd sa place, à tout seigneur tout honneur, bonne renommée vaut mieux que ceinture dorée ${ }^{1}$, etc. Il arrive aussi que le français combine la rime et l'allitération: chat et chaton chassent le raton; argent fait rage, amour, mariage, etc.

L'anglais, pour lequel la rime n'est pas la préoccupation primordiale, n'en offre pas moins quelques exemples de rimes riches: there's many a slip twixt the cup and the lip (il y a loin de la coupe aux lèvres), joy and sorrow are today and tomorrow (les joies et les peines sont de tous temps), in times of prosperity friends are plenty (on connaît le véritable ami dans le besoin), no bees no honey, no work no money (toute peine mérite salaire), etc., à côté d'une majorité d'autres: no gains without pains (on n'a rien sans peine), east, west, home's best (on n'est nulle part mieux que chez soi), good health is above wealth (il n'est trésor que de santé), for ill do well, then fear no hell (rendre le bien pour le mal), he gives twice who gives in a trice (c'est obliger deux fois qu'obliger promptement) ${ }^{2}$, etc.

\section{Formes allitérées}

Bien que l'allitération appartienne surtout au génie des langues septentrionales, le français présente un grand nombre de dictons fondés sur ce procédé: un coup de langue est pis qu'un coup de lance, selon ta bourse gouverne ta bouche, qui se sent morveux se mouche, il n'y a pas de fumée sans feu, faites-vous miel et les mouches vous mangeront, on ne peut vouloir la poule et les poussins, etc.

L'anglais, a fortiori, y recourt encore davantage: $\underline{b}$ etter bend than break (mieux vaut plier que rompre), fair feathers make fair fowls (les belles plumes font les beaux oiseaux), where there's a will there's a way (qui veut la fin veut les moyens), spare the rod and spoil the child (qui aime bien châtie bien), more fools, more fun (plus on est de fous, plus on rit), seldom seen, soon forgotten (loin des yeux, loin du cœur), cut your coat according to your cloth (il faut tailler la robe selon le drap), lovers live by love as larks by leeks (les amoureux vivent d'amour et d'eau claire), speech is silver, silence is gold (la parole est d'argent, le silence est d'or), time and tide wait for no man (le temps perdu ne revient pas), we never know the worth of water till the well is dry (bien perdu, bien connu), etc. 


\section{DICTONS MÉTÉOROLOGIQUES}

De tout temps, l'homme a fixé dans des dictons rimés les rapprochements entre événements naturels et comportement animal par lesquels il prétendait expliquer ou prédire les changements climatiques.

Dans ce domaine aussi, le français fait parfois appel à la rime riche: le soleil de la Chandeleur annonce l'hiver et malheur; chante la grive, la pluie arrive; quand le coq chante à midi, signe d'un temps de paradis, etc., en la combinant même avec l'allitération: les mouches et les taons piquent avant le mauvais temps; si les souris $\underline{s}$ 'agitent et les rats, la pluie viendra. Mais ce sont les rimes pauvres qui constituent la majorité: Noël au balcon, Pâques aux tisons; araignée tissant, mauvais temps; ciel pommelé et femme fardée ne sont pas de longue durée; la carpe saute, de l'eau sans faute; quand l'hirondelle vole à terre, adieu la poussière; quand le chat se débarbouille, le temps se brouille; un jour est mouillé, l'autre sec, quand le coucou ouvre son bec; quand le coq chante avant la nuit, signe de pluie; en avril ne te découvre pas d'un fil, en mai, fais ce qu'il te plaît; pluie de février vaut fumier, etc.

Langlais n'est pas en reste mais se contente de rimes pauvres: when the crows flock together, prepare for wintry weather (quand les corneilles s'assemblent, du bois pour ton hiver assemble); when the flies sting, rain they'll bring (les mouches et les taons piquent avant le mauvais temps); till April is dead, change not a thread (en avril, ne te découvre pas un fil); if the cock goes crowing to bed, he's sure to rise with a watery head (quand le coq chante le soir, c'est signe qu'il va pleuvoir); a mackerel sky is never long dry, a mackerel sky and mare's tails make lofty ships carry low sails (ciel pommelé et nuages effilés annoncent grand vent), etc.

\section{LOCUTIONS}

Les locutions basées sur la rime ou l'allitération peuvent être de nature diverse: verbale, adjectivale, adverbiale.

\section{Formes rimées}

Curieusement, le français ne forme que peu de locutions de cette sorte, qu'elles soient verbales (avoir son genou dans le cou, être mou du bout, payer en chats et en rats, faire mallette et paquette à qqn), adjectivales (sage comme une image, pédoque comme un phoque, margouillat sur le tas) ou adverbiales (à la Saint-Glin-Glin), etc.

L'anglais, mais c'est pour lui normal, n'est pas plus riche, avec des louctions verbales comme to have ants in one's pants (être très agité), to take the cake (remporter la palme), to toil and moil (peiner, suer sang et eau), to bring haddock to the paddock (tout perdre), to gallop one's antelope (se masturber), des locutions adjectivales comme full as a bull (ivre), loose as a goose (libertine), snug as a bug in a rug (bien aise, confortable), des locutions adverbiales comme by hook and by crook (d'une manière ou d'une autre, coûte que coûte), etc.

\section{Formes allitérées}

Tout aussi bizarrement, le français rivalise ici avec l'anglais, tant les locutions allitératives y abondent. 
Ainsi les locutions verbales, $\underline{b} a t t r e$ le $\underline{b} e u r r e, \underline{b} a t t r e ~ l a ~ b r e l o q u e, ~ \underline{m a n g e r ~ l e ~} \underline{\text { mor}}-$ ceau, bouffer des briques, promettre monts et merveilles, ne pas mâcher ses mots, payer les pots cassés, avoir du vent dans les voiles, se parer des plumes du paon, etc., parfois associées à la rime comme dans chercher des cheveux sur des œufs, peuventelles se comparer aux anglaises to beat about the bush (tourner autour du pot), to beat black and blue (rosser), to sleep like a log (dormir profondément), to shake in one's shoes (trembler de peur), to have bats in the belfry (avoir l'esprit dérangé), to have neither chick not child (n'avoir pas de progéniture), to set all at sixes and sevens (mettre la pagaille), to find fish on one's fingers (invoquer une excuse), etc.

Les locutions adjectivales, moins nombreuses en français, comprennent blond comme les blés, bouche bée, léger comme l'oiseau St-Luc, empêtré comme une poule à trois poussins, seç comme un coup de trique, etc., tandis que l'anglais offre safe and sound (sain et sauf, forme également allitérée en français), too big for one's boots (arrogant), neither fish nor fowl (ni chair ni poisson), dressed up like a dog's dinner (chic, élégant), hung like a horse (doté d'un bel appareil sexuel), in full feather (en grande toilette), born and bred (pur sang, authentique), bitten by a barnmouse (saoul), etc., auxquelles il convient d'ajouter des locutions comparatives comme as blind as a bat (aveugle, myope), as busy as a bee (très affairé), as fit as a flea (frais et dispos), as proud as a peacock et as proud as a dog in a doublet (fier comme un paon), as British as beef (britannique jusqu'au bout des ongles), as dead as a doornail (mort et bien mort), as pretty as a picture (jolie comme un cœur), as merry as mice in malt (plein d'entrain, joyeux), etc.

Et l'on peut de même aligner les locutions adverbiales françaises à tort et à travers, pieds et poings liés, au petit poil, montre en main, bras dessus bras dessous, comme du poisson pourri, etc., face aux anglaises head over heals (éperdument), from top to toe (de la tête aux pieds), with a wave of the wand (d'un coup de baguette magique), etc.

\section{EXCLAMATIONS, APOSTROPHES ET JURONS}

L'imaginaire collectif a souvent eu recours à la rime et à l'allitération pour créer des exclamations plaisaintes et des jurons fantaisistes.

\section{Formes rimées}

Selon les situations, le français peut ainsi s'écrier Dieu vous bénisse et vous fasse le nez comme j'ai la cuisse! (souhait à qui vient d'éternuer); écrivez, greffier ! (invitation à noter quelque chose); un peu, mon neveu ! (et comment!), tu l'as dit, bouffí! (c'est l'évidence même), tu me le copieras sur du papier de soie! (marque d'étonnement), etc; l'anglais s'exclamera fuck a duck! (ben alors là!), jeepers creepers! (sapristi!), hell's bells! (sacrebleu!), hot-diggity-doggity! (mazette!), pody cody! (saperlipopette!), etc.

Dans le même esprit, la tendance ludique de l'individu à manipuler les mots en dehors des cadres conventionnels a donné naissance à des formules d'apostrophe ou d'interpellation où la rime ne remplit qu'une fonction explétive. Ainsi, en français: tu vas me le payer, Aglaé !, fonce, Alphonse, à la revoyure, Arthur !, à l'aise, Blaise!, à la tienne, Étienne!, recule, Hercule!, relaxe Max, cool, Raoul, etc; en anglais: see 
you later, alligator! (à plus tard!), in a while, crocodile (un moment!), give me some skin, Flynn (tope-là!), pass me the sherbert, Herbert (passe-moi l'objet!), like your tail, nightingale! (salutation à une jolie fille), etc.

\section{Formes allitérées}

Ce type d'exclamation est rare en français: adieu, veau, vache, cochon, couvée! (adieu les illusions), cric-croc! (à ta santé!), vertu de ma vie! (juron bénin), mais particulièrement fréquent en anglais: blue-bottles (crénom!), Christ on a crutch! (sacristi!), drop dead! (allez au diable!), Good Godfrey (bon Dieu!), good gracious! (bonté divine!), good grief! (miséricorde), goody goody gumdrop! (nom d'un petit bonhomme!), great guns! (nom d'un tonnerre!), holy jumping Mother of Jesus! (Jésus, Marie, Joseph!), jumping Jehoshaphat! (sapristi!), jumping Jew's harp! (sacrebleu!), Lord love a duck! (mince alors!), please the pigs! (plaise à Dieu!), suffering saints! (par tous les saints!), suffering snakes! (id.), suffering seaserpents! (id.), suffering seaweeds! (id.), etc.

\section{SUBSTANTIFS ET VERBES}

La rime et l'allitération interviennent dans quantité de mots composés, utilisés parfois aussi comme verbes ou éléments de locutions verbales. Il peut s'agir de mots obtenus (1) par le redoublement d'un élément dénué de sens pour former la rime ou l'allitération, (2) par l'apposition de deux éléments pourvus de sens rimant ou allitérant l'un avec l'autre.

\section{Redoublements}

\section{Formes rimées}

Le mot peut se composer d'un son, d'une syllabe répétés tels quels. Ainsi, en français: dare-dare, digue-digue (et tomber en digue-digue), fri-fri, fute-fute, kif-kif, minamina, mita-mita (et faire mita-mita), faire niq-niq, pia-pia, pouet-pouet, soua-soua, tchik-tchik, teuf-teuf, tsoin-tsoin, faire zig-zig, et les formes sans trait d'union: baba (et rester baba), bébé, bibi, blabla, bobo, bouiboui, caca (et faire caca), cancan, chichi (et faire des chichis), clicli, coco, coucou, cracra, cricri, cucu, dada (et enfourcher son dada), didi, dodo (et faire dodo), dondon, fifi, flafla, flonflon, froufrou, gaga (et devenir gaga), glagla, glouglou, gnangnan, gogo, grigri, jaja, jinjin, jojo, kiki (et serrer le kiki à qqn), lolo, lulu, mémé, mimi (et faire mimi), nana, néné, neuneu, nunu, papa, pépé, pépée, pioupiou, pipi (et faire pipi), ronron, tata, titi, toctoc, tonton, toto, toutou, tutu, zinzin, zizi, zozo (et faire le zozo). En anglais: blah-blah (bavardage creux, blabla), bobo (ivre), booboo (blessure, bobo; gaffe), booboos (testicules), buddy-buddy (copains), chop-chop (vite, dare-dare), dee-dee (saoul), fig-fig (coï), to play footyfooty (faire du pied), gaga (gâteux, gaga), gee-gee (cheval, dada), goody-goody (prude), hush-hush (très secret), jig-jig (coï), lulu (chose ou personne remarquable), she-she (jeune femme), so-so (moyen, comme ci comme ça), yak-yak (bavardage, blabla), zig-zig (coït, zig-zig), etc.

Le mot peut se composer d'un son, d'une syllabe répétés en modifiant leur consonne initiale ou en les faisant précéder d'une consonne ou d'une syllabe supplé- 
mentaire, comme en français, pêle-mêle, ramdam, d'une part, radada, raplapla, $\underline{\text { rififi, }}$ rikiki, zigouigoui, d'autre part; en anglais, les exemples sont beaucoup plus nombreux, tels boogily-woogily (en désordre), to boohoo (pleurer abondamment), bowwow (chien, toutou) et to bow-wow (aboyer), ducky-wwucky (merveilleux, super), fancy-schmancy (sophistiqué), to play footsie-wootsie (faire du pied), fuddy-duddy (vieille baderne) et to fuddy-duddy (agir sottement), hanky-panky (supercherie), helter-skelter (pêle-mêle), hocus-pocus (mystification), hoity-toity (fier, snob), hootchie-cootchie (danse du ventre et danseuse), hustle-bustle (coït), lovey- $\underline{\text { dovey }}$ (mon petit chou), nitty-gritty (le fin d'une affaire), rantan (nouba) et to go on the rantan (faire la nouba), razzle-dazzle (confusion, pagaille), roly-poly (grassouillet), rum(crise d'épilepsie, digue-digue; crise de colère, rouspétance), dans le premier cas; itsy-bitsy (minuscule, rikiki), okey-dokey (d'accord), ooly-drooly (amours juvéniles), the hoi polloi (la foule, les masses), etc. dans le second cas.

Le français possède encore une forme dans laquelle il ajoute une terminaison à l'élément redoublé, ce qui annule la rime et ne laisse que l'allitération : bébète, bibiche, bibine, bloblote, bobonne, cacade, cacasse, chochotte, cocotte, dodoche, doudoune, fifine, foufoune, gnognote, guiguite, mémère, moumoute, nénette, nunuche, pépère, pépette, popote, popotin, tétère, titine, totoche, tutute, etc; c'est le procédé qu'il utilise aussi pour former des verbes à partir de redoublements rimés : blablater, cancaner, chouchouter, cocoter, glaglater, glouglouter, ronronner, etc.

\section{Formes allitérées}

Si, dans l'élément redoublé, ce n'est pas la consonne initiale mais la voyelle du radical qui change, il en résulte une allitération. Ainsi trouve-t-on en français: couci-couça, cric-croc, flic-flac, flipp-flop, fric-frac, frotti-frottạ, méli-mélo, mic-mac, prêchiprêcha ; bique et bouc,

bric-à-brac, de brịc et de broc, et patati et patata, ric à racc, etc.; en anglais : chitchat (bavardage), dilly-dally (perdre son temps), ding-dong (cloche; engueulade), dingledangle (pénis), fiddle-faddle (balivernes), flim-flam (boniments; mystification) et to flím-flam (duper), flịp-flạp (pénis), flịp-flopp (saut périlleux), jịm-jạms (nervosité, frousse, trac), mish-mash (mélange, fatras), niggers-noggers! (juron), tịp-top (excellent), tittle-tattle (commérages) et to tittle-tattle (bavarder, cancaner), wiggle-waggle (balancer) et to wigggle-wagggle (bavarder), wịshy-washy (fade; faible), etc.

\section{Appositions}

\section{Formes rimées}

Ce type de syntagme, qui associe deux éléments pourvus de sens rimant ensemble, ne paraît guère tenter le français, si ce n'est par redoublement d'un même élément: bonbon, chouchou, coupe-coupe, crincrin, donnant donnant, fil-à-fil, tour de passepasse, pousse-pousse, soin-soin, train-train, etc. ; l'anglais, au contraire, l'affectionne: beaver retriever (libertin), culture vulture (amateur de culture), dizzy Lizzie (fille stupide), fat cat (financier d'un parti politique), frame dame (jolie fille, beau châssis), glad pad (endroit plein d'ambiance), gruesome, twosome (deux personnes inséparables), handsome ransom (somme rondelette), hen pen (collège de filles), hot shot 
(jeune ambitieux; scène osée dans un film), hot spot (situation fâcheuse, boîte de nuit), jug mug (prisonnier), khaki-wacky (folle de militaires), lead-head (crétin), legal eagle (avocat), lush mush (joli minois; mets délicieux), plain Jane (laideron), rabbit habit (coï), rough stuff (brutalité), silly billy (nigaud), skunk-drunk (saoul), slick chick (fille à la page), slim Jim (grand échalas), tricky Vicky (femme de tête), etc.

Dans l'argot rimé, qui est propre à la langue anglaise, le procédé est également très prisé: army and navy (pour gravy, sauce), bows and arrows (pour sparrows, moineaux), Dover Harbour (pour barber, coiffeur), frog a $\underline{\log }$ (pour $\underline{\mathrm{dog}}$, chien), hickey-hockey (pour jockey), mutter and stutter (pour butter, beurre), rub-a-dub (pour club ou pub), umble-cum-stumble (pour rumble, deviner, subordorer et tumble, comprendre, piger), whale and gale (pour jail, prison), etc.

\section{Formes allitérées}

Le français semble porté davantage vers ce genre de syntagmes, dont beaucoup relèvent du langage non conventionnel: beau blond, bleubite (simple recrue), casse-croûte (collation légère), casse-couilles (importun), coup de collier (effort intense), mangemerde (avare), mannequin à machabées (corbillard), marchand de mort subite (mauvais médecin), mère maqua (patronne de maison close), mie de pain mécanique (puce), petit pain, pisse-copie (journaliste), perruque en peau de fesses (crâne chauve), poudre de perlimpinpin (remède de charlatan), shampooing à Charles le Chauve (fellation), tasse à thé (urinoir public), etc.

L'anglais, friand d'allitérations, n'est évidemment pas en reste: belly button (nombril), busybody (mouche du coche), big bug (personnage important), cocky customer (effronté), cow college (institut agronomique), crotch cobra (pénis), dead duck (échec), flat fish (lourdaud, empoté), flea flint (avare), green goose (prostituée), lounge lizard (gigolo), lovebird look (regard énamouré), double-dealer (faux jeton),

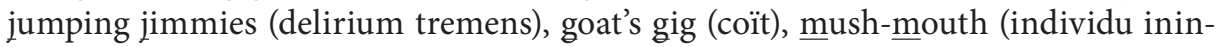

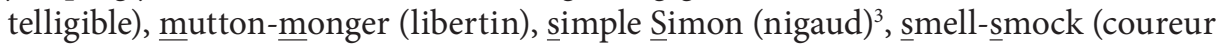
de jupons), etc.

Et il applique aussi largement le procédé dans son argot, rimé, où l'apposition de termes peut reposer (1) sur une synonymie: borrow and beg (pour egg, œuf), coals and coke (pour broke, démuni), fun and frolics (pour ballocks, testicules), grumble and grunt (pour cunt, sexe de la femme), hoot and holler (pour dollar), pillar and post (pour ghost, fantôme), pudding and pie (pour eye, œil), shake and shiver (pour

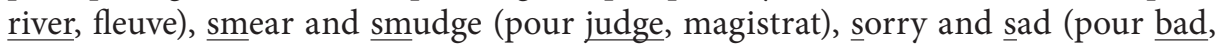
mauvais), stammer and stutter (pour butter, beurre), struggle and strife (pour wife, épouse), trolly and truck (pour fuck, coï), twist and twirl (pour girl, fille); (2) sur une association d'idées: ball and bat (pour hat, chapeau), bottle of beer (pour ear, oreille), bread and butter (pour gutter, rigole), cash and carried (pour married, mariés), cat and kitties (pour titties, seins), collar and cuff (pour puff, homme efféminé), cow and calf (pour laugh, rire), cuddle and kiss (pour miss, jeune fille), dot and dash (pour moustache), fiddle and flute (pour suit, costume), frock and frill (pour chill, rhume et ill, malade), horse's hoof (pour poof, homme efféminé), lion's lair (pour chair, chaise), peaches and pears (pour stairs, escalier), pot and pan (pour old man, vieillard), satin and silk (pour milk, lait), tea and toast (pour post, poteau d'arrivée), read and write (pour fight, dispute); (3) sur une opposition: pleasure and pain (pour 
$\underline{\text { rain, }}$ pluie), raw and ripe (pour pipe), saint and sinner (pour dinner), ou encore (4) sur l'arbitraire le plus absolu: balmy breeze (pour cheese, fromage), chevy chase (pour face, visage), dinky-dirt (pour shirt, chemise), dirty daughter (pour water, eau), down the drains (pour brains, cerveau, intelligence), garlic and glue (pour stew, émoi, bouleversement), jam-jar (pour car, voiture), lean and lake (pour steak), mad Mick (pour prick, pénis), pickled pork (pour talk, parler), seldom seen (pour limousine),

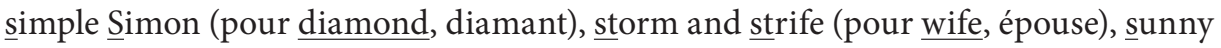

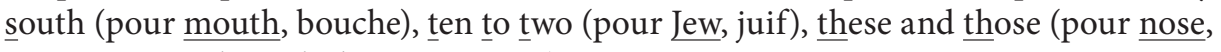
nez, toes, orteils et clothes, vêtements), etc.

\section{NOTES}

1. Que l'anglais traduit par une forme allitérée: a good fame is better than a good face.

2. Que le français traduit par une forme allitérée: qui donne tôt donne deux fois.

3. À ne pas confondre avec l'argot rime simple Simon (pour diamond, diamant).

\section{RÉFÉRENCES}

Augé, P. et al. (1960): Grand Larousse encyclopédique, Paris, Larousse.

Аyто, J. (1999): The Oxford Dictionary of Slang, Oxford, Oxford University Press.

Berry, L. V. and M. van den Bark (1953): The American Thesaurus of Slang, New York, Crowell.

Browning, D. C. (1969): Dictionary of Quotations and Proverbs, London, Everyman.

Brunet, F. and D. McCavana (1996): Dictionnaire bilingue de l'argot d'aujourd'hui, Paris, Pocket-Langues pour tous.

Cellard, J. et A. Rey (1991): Dictionnaire du français non conventionnel, Paris, Hachette.

Colin, J. P. et J. P. MÉvel (1992): Dictionnaire de l'argot, Paris, Larousse.

DeAK, E. (1957): Grand dictionnaire d'américanismes, Paris, Ed. du Dauphin.

DE Dony, Y. (1951): Léxico del lenguaje figurado, Buenos Aires, Desclée.

Duneton, C. (1990): Le Bouquet des expressions françaises, Paris, Le Seuil.

FrankLin, J. (1996): A Dictionary of Rhyming Slang, London, Routledge.

Leitner, M. J. and J. R. Lanen (1965): Dictionary of French and American Slang, New York, Crown Publications.

Little, W. et al. (1969): Shorter Oxford Dictionary, Oxford, Oxford University Press.

Maloux, M. (1960): Dictionnaire des proverbes, sentences et maximes, Paris, Larousse.

Mansion, J.E. (1980): Harrap's French-English and English-French Dictionary, London, Harrap.

Montreynaud, F. et al. (1994): Dictionnaire des proverbes et dictons, Paris, Le Robert.

Partridge, E. (1961): A Dictionary of Slang and Unconventional English, London, Routledge and Keagan Paul.

Rey, A. et S. Chantreau (1997): Dictionnaire des expressions et locutions, Paris, Le Robert.

SAndry, G. et M. Carrère (1957): Dictionnaire de l'argot moderne, Paris, Ed. du Dauphin.

Spears, R.A. (1991): A Dictionary of Slang and Euphemism, New York, Penguin Books.

Wentworth, H. and S. B. Flexner (1960): Dictionary of American Slang, New York, Crowell. 